\title{
Tendência da mortalidade por câncer de colo uterino em Cáceres - Mato Grosso
}

\section{(2012 - 2019)}

\author{
Cervical cancer mortality trends in Caceres - Mato Grosso (2012 - 2019) \\ Tendencia de la mortalidad por cáncer de cuello uterino en Cáceres - Mato Grosso (2012 - 2019)
}

Max Paulo Rocha Leite ORCID: https://orcid.org/0000-0001-8168-0770 Universidade do Estado do Mato Grosso, Brasil

E-mail:max.paulo@unemat.br

Bianca Teshima de Alencar ORCID: https://orcid.org/0000-0001-6812-3494 Universidade do Estado do Mato Grosso, Brasil

E-mail: bianca.teshima@unemat.br

Lúbia Maieles Gomes Machado

ORCID: https://orcid.org/0000-0003-4538-5138 Universidade do Estado do Mato Grosso, Brasil E-mail: lubia.machado@unemat.br

Stefany Caroliny de Souza

ORCID: https://orcid.org/0000-0001-5469-4659 Universidade do Estado do Mato Grosso, Brasil E-mail: stefany.caroliny@unemat.br

Nayara Ferreira Carvalho ORCID: https://orcid.org/0000-0001-7528-2821

Centro Universitário Alfredo Nasser, Brasil E-mail: nayferreira_go@hotmail.com

Priscila Campos Santos

ORCID: https://orcid.org/0000-0002-6400-4129 Universidade do Estado do Mato Grosso, Brasil E-mail: priscila.santos@unemat.br

Debora Pereira dos Santos

ORCID: https://orcid.org/0000-0001-5738-770X Universidade do Estado do Mato Grosso, Brasil E-mail: debora.pereira@unemat.br

Jaýne Santos Borges

ORCID: https://orcid.org/0000-0003-1070-8101 Universidade do Estado do Mato Grosso, Brasil E-mail: jayne.borges@unemat.br

Hellen Catharine Silva Batista ORCID: https://orcid.org/0000-0002-2563-3109 Universidade do Estado do Mato Grosso, Brasil E-mail: hellenbatista@yahoo.com.br

Natasha Rayane de Oliveira Lima ORCID: https://orcid.org/0000-0002-7238-8476 Universidade do Estado do Mato Grosso, Brasil E-mail: natasha.rayane@unemat.br

\begin{abstract}
Resumo
Objetivo: evidenciar a tendência da mortalidade por câncer de colo uterino no município de Cáceres e no estado de Mato Grosso no período de 2012 a 2019. Metodologia: Trata-se de um estudo descritivo, retrospectivo e transversal dos óbitos por neoplasia de câncer de colo uterino, notificados no período de 01 de janeiro de 2012 a 31 de dezembro de 2019 em Cáceres, Mato Grosso, Brasil. Resultados e Discussão: Dos dados analisados, o grupo etário de 60 a 69 anos foi o que apresentou o maior número de óbitos durante o período estudado, e a cor/raça parda foi a que mais se destacou entre os óbitos para o câncer de colo uterino. Foi identificada uma tendência de estabilidade nas taxas de mortalidade por câncer do colo do útero em Cáceres-MT. Conclusão: Acredita-se que os resultados tenham influência de fatores socioeconômicos e das políticas públicas de saúde a mulher no país. Desse modo, ressalta-se a importância da ampliação de ações voltadas a saúde da mulher com a finalidade da redução dos óbitos por patologia altamente evitáveis como o câncer do colo do útero.
\end{abstract}

Palavras-chave: Neoplasias do colo do útero; Saúde da mulher; Neoplasmas; Mortalidade. 


\begin{abstract}
Objective: to highlight trends of cervical cancer mortality in the municipality of Cáceres and in the state of Mato Grosso in 2012 to 2019. Methodology: This is a descriptive, retrospective and cross-sectional study of deaths from uterine cancer neoplasms, reported from January 1, 2012 to December 31, 2019 in Cáceres, Mato Grosso Brazil. Results and Discussion: From the data analyzed, the age group of 60 to 69 years was the one that presented the highest number of deaths during the study period, and the brown color/race was the one that stood out most among deaths for cervical cancer. A stable trend in cervical cancer mortality rates was identified in Cáceres-MT. Conclusion: It is believed that the results have an influence of socioeconomic factors and of public health policies to women in the country. Thus, it is important to expand actions aimed at women's health to reduce deaths from highly preventable pathologies such as cervical cancer.
\end{abstract}

Keywords: Cervical neoplasms; Women's health; Neoplasms; Mortality.

\title{
Resumen
}

Objetivo: evidenciar la tendencias de la mortalidad por cáncer de cuello uterino en el municipio de Cáceres y en el estado de Mato Grosso en el período de 2012 a 2019. Metodología: Se trata de un estudio descriptivo, retrospectivo y transversal de los óbitos por neoplasia de cáncer uterino, notificados en el período del 01 de enero de 2012 al 31 de diciembre de 2019 en Cáceres, Mato Grosso Brasil. Resultados y Discusion: De los datos analizados, el grupo de edad de 60 a 69 años fue el que presentó el mayor número de óbitos durante el período estudiado, y el color/raza parda fue el que más se destacó entre los óbitos para el cáncer de cuello uterino. Se identificó una tendencia de estabilidad en las tasas de mortalidad por cáncer de cuello uterino en Cáceres-MT. Conclusión: Se cree que los resultados tengan influencia de factores socioeconómicos y de las políticas públicas de salud a la mujer en el país. De ese modo, se resalta la importancia de la ampliación de acciones volcadas a la salud de la mujer con la finalidad de la reducción de los óbitos por patología altamente evitables como el cáncer del cuello del útero.

Palabras clave: Neoplasias del cuello uterino; Salud de la mujer; Neoplasmas; Mortalidad.

\section{Introdução}

O câncer de colo uterino é um tipo de carcinoma originado da replicação desordenada do epitélio que reveste o útero podendo comprometer estruturas adjacentes. Geralmente essa proliferação desordenada é provocada por lesões ocasionadas pela infecção persistente de alguns tipos oncogênicos do HPV - Papilomavírus Humano (Brasil, 2013). O câncer do colo do útero é uma das doenças mais graves à saúde da mulher, chegando a representar mundialmente o quarto tipo de câncer com maior incidência no sexo feminino. Estimativas da Organização Mundial de Saúde (OMS) de 2018, apontaram cerca de 570 mil novos casos, e mais de 311 mil óbitos por câncer do colo do útero em todo o mundo (WHO, 2020).

De acordo com a Organização Pan-Americana da Saúde (OPAS), aproximadamente 90\% das estimativas relacionadas aos óbitos pela doença, ocorreram em países de baixa e média renda, principalmente em países localizados na América Central e na do Sul. Acredita-se que as taxas de mortalidade por câncer de colo do útero são três vezes mais altas na América Latina e no Caribe do que na América do Norte devido as desigualdades socioeconômicas, de gênero e do acesso aos serviços de saúde (PAHO, 2016; WHO, 2020; INCA, 2021a).

O Instituto Nacional de Câncer (INCA, 2021a) apontou que no ano de 2019 ocorreram 6.596 óbitos por câncer do colo uterino no Brasil, correspondendo a uma taxa ajustada de mortalidade de 5,33 casos a cada 100 mil mulheres. E estimaram para 2020, o registro de 16.710 novos casos com um risco estimado de 15,38 casos a cada 100 mil mulheres. Referente aos registros de óbitos por regiões do Brasil, as maiores incidências de morte por câncer de colo do útero a cada 100 mil habitantes, ocorrem na região Norte (26,24 óbitos), região Nordeste (16,10 óbitos), seguido da região Sul (12,60 óbitos), região Centro-Oeste (12,35 óbitos) e Sudeste (8,61 óbitos).

O Câncer de colo do útero consiste em uma doença de progressão lenta e com fase pré-clínica silenciosa (sem sintomas). Sua detecção pode ocorrer por exames clínicos, laboratoriais ou radiológicos, em pessoas com ou sem sinais e sintomas sugestivos da doença. (INCA, 2021b). No âmbito do Sistema Único de Saúde (SUS), o método de rastreamento do câncer do colo do útero e das suas lesões precursoras é a realização do exame citopatológico oncótico, conhecido como Papanicolau, sendo preconizado as coletas anualmente em mulheres na faixa etária de 25 a 64 anos de idade que já tiveram atividade sexual (INCA, 2011). Com relação ao tratamento do câncer de colo de útero, os métodos utilizados variam entre 
cirurgia, quimioterapia ou radioterapia, sendo o melhor método elegido de acordo com o estágio de evolução da doença, idade, entre outros fatores (INCA, 2021b).

Considerando a magnitude do câncer do colo de útero e os seus fatores associados, entende-se a importância em observar os indicadores de saúde relativos a esta patologia não apenas em escalas globais, como também por regionalidades. Quando dados levantados são trabalhados geram-se indicadores de saúde que possibilitam mensurar a dimensão de saúde de uma população alvo, por exemplo, ao se trabalhar registros de óbitos por 100 mil habitantes, em determinado espaço geográfico e ano, é gerado a taxa de mortalidade (PAHO, 2018; RIPSA, 2008). O indicador da taxa de mortalidade por neoplasias permite estimar o risco de morte por neoplasias malignas e dimensionar a magnitude da doença como problema de saúde pública (RIPSA, 2008). Nesse sentido, este trabalho tem como objetivo principal evidenciar a tendência da mortalidade por câncer de colo uterino no município de Cáceres e no estado de Mato Grosso no período de 2012 a 2019.

\section{Metodologia}

Trata-se de um estudo descritivo, retrospectivo e transversal dos óbitos por neoplasia de câncer do colo do útero, notificados no período de 01 de janeiro de 2012 a 31 de dezembro de 2019 em Cáceres, Mato Grosso Brasil.

A coleta de dados foi realizada a partir de dois bancos de informação, para os dados de óbitos por câncer do colo do útero ou demais neoplasias foi utilizado o SIM - Sistema de Informação sobre Mortalidade (DATASUS, 2020b). O SIM é um dos componentes do Sistema de Informação em Saúde, e foi criado pelo Departamento de Informática do Sistema Único de Saúde (DATASUS), que utiliza como fonte de informação a Declaração de Óbito. Esse sistema é útil para a obtenção de dados sobre mortalidade, podendo servir como base de orientação de planejamentos e ações em saúde pública (Alves et al., 2009). Alguns problemas interferem na qualidade dos dados, como a subnotificação e o número de óbitos com causa mal definida (Ferlay et al., 2015). Para tal, foram considerados os registros ocorridos entre 2012 a 2019, classificados como C53. Neoplasia maligna do colo do útero ou as demais neoplasias classificadas no capítulo II. Neoplasmas [tumores], conforme a $10^{\mathrm{a}}$ Classificação Internacional de Doenças (CID-10).

A população foi obtida por meio da estimativa da população feminina residente por ano, segundo município distribuída por faixa etária e sexo, através da projeção proveniente do IBGE - Instituto Brasileiro de Geografia e Estatística (DATASUS, 2020a). Utilizou-se como critérios de elegibilidade: Ser do sexo feminino; residir em Cáceres; e, óbito por câncer de colo uterino ou demais neoplasias ter ocorrido entre 01 de janeiro de 2012 a 31 de dezembro de 2019.

A taxa de mortalidade por causa específica para o município de Cáceres e para o estado de Mato Grosso foi calculada pela divisão entre o total de óbitos por câncer do colo do útero ou ocorrido pelas demais neoplasias, sobre a estimativa da população feminina estudada, por ano. Nos cálculos das taxas, os coeficientes foram multiplicados pela constante 100.000, como apresentado na equação abaixo:

$$
\text { Taxa }=\frac{\text { Número de óbitos de residentes por neoplasias malignas }}{\text { População total residente ajustada para o meio do ano }} x 100.000
$$

Os dados foram organizados e armazenados em planilha no software Microsoft Excel®, as tabelas e as variáveis categóricas foram estimadas as frequências absoluta e relativa no mesmo programa. Com a finalidade de verificar alteração temporal na ocorrência de aumento ou redução das taxas de mortalidade padronizadas foi determinada a variação percentual relativa (VPR). A VPR é igual a última observação da série menos o valor observado para o ano de início dividido pelo valor observado para o ano de início multiplicado por 100 (Silva et al., 2008). 
Para análise de tendência foi utilizado o programa estatístico R versão 4.1.1 por meio das bibliotecas "Imtest", "prais" e “ggplot2”, trata-se de um software gratuito de livre acesso para realização de gráficos e estatísticas, podendo ser utilizado em uma variedade de plataformas. Modelos de regressão linear foram utilizados para análise de tendência, cujas variáveis dependentes foram as taxas(Y) e o tempo em anos como variável independente (X). Para evitar autocorrelação entre os termos da equação de regressão foi aplicada a transformação da variável "ano" em ano-centralizado (ano menos o ponto médio do período de estudo). As tendências foram interpretadas como crescente ( $<<0,05$ e $\beta 1$ positivo); decrescente ( $p<0,05$ e $\beta 1$ negativo) e estável ( $\mathrm{p} \geq 0,05)$ (Antunes \& Cardoso, 2015).

O estudo foi realizado por meio dos dados disponíveis em bases de dados secundários de acesso livre, os quais não apresentam variáveis que possibilitam a identificação de indivíduos, de forma que não há necessidade de aprovação pelo Comitê de ética em pesquisa, conforme estabelece a Resolução no 466 de 12 de dezembro de 2012 e Resolução 510 de 2016 do conselho nacional de saúde.

\section{Resultados}

Entre 2012 a 2019, ocorreram 31 óbitos por câncer do colo uterino, com uma média de aproximadamente 04 mortes anuais na população feminina de Cáceres-MT. Dentro dos grupos etários analisados o grupo etário com o menor número de óbitos por esta causa foi de 20 a 29 anos com 02 (6,45\%) óbitos durante os oito anos estudado, o grupo etário de 60 a 69 anos foi o que apresentou o maior número de óbitos sendo 09 (29.03\%) óbitos durante o período estudado. Não ocorreu registros de óbitos em idades inferiores a 20 anos. E foi observado que a cor/raça parda apresentou 22 (70,96\%) óbitos na série estudada para o câncer de colo uterino, seguido da raça branca $(22,58 \%)$ em a raça preta com $(6,45 \%)$ óbitos (Tabela 1$)$.

Tabela 1. Frequência absoluta (N) e relativa (\%) de óbitos de acordo com a faixa etária e cor/raça por neoplasia do colo do útero do município de Cáceres- MT, entre 2012 a 2019.

\begin{tabular}{ccc}
\hline Grupo etário & $\mathbf{N}$ & $\mathbf{\%}$ \\
\hline $20-29$ & 02 & 6,45 \\
$30-39$ & 05 & 16,13 \\
$40-49$ & 03 & 9,68 \\
$50-59$ & 04 & 12,90 \\
$60-69$ & 09 & 29,03 \\
$70-79$ & 05 & 16,13 \\
$\geq 80$ anos & 03 & 9,68 \\
\hline Cor/raça & $\mathbf{N}$ & $\mathbf{\%}$ \\
\hline Branca & 07 & 22,58 \\
Preta & 02 & 6,45 \\
Parda & 22 & 70,97 \\
\hline
\end{tabular}

Fonte: Autores (2021).

O município de Cáceres no ano de 2014 obteve o maior número de óbitos na série estudada com 06 (19,35\%) para neoplasias do colo uterino, as taxas se apresentaram oscilantes durante o período estudando com uma VPR de 27,12\%, o estado de Mato Grosso apresentou o maior registro em 2019 com 105 (15,04\%) óbitos, as taxas se apresentaram crescentes e oscilantes durante o período estudado apresentando uma VPR de 36,38\%. Nas demais neoplasias o maior registro para o município de Cáceres foi em 2016 e 2017 com 40 (16,19\%) óbitos consecutivos, o estado de Mato Grosso apresentou o maior registro em 2017 com 787 (13,99\%) óbitos. Ressalta-se que apesar do estado ter apresentado números de óbitos superior ao município de Cáceres durante os 08 anos estudados, as taxas de mortalidades especificas do município se mantiveram em toda série, superior as taxas do estado (Tabela 2). 
Tabela 2. Taxa de mortalidade e variação percentual por neoplasias para a população feminina do município de Cáceres e estado de Mato Grosso, entre 2012 a 2019.

\begin{tabular}{|c|c|c|c|c|c|c|c|}
\hline \multirow{11}{*}{ 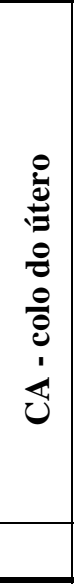 } & & \multicolumn{3}{|c|}{ Cáceres } & \multicolumn{3}{|c|}{ Mato Grosso } \\
\hline & Ano & $\mathbf{N}$ & $\%$ & Taxa & $\mathbf{N}$ & $\%$ & Taxa \\
\hline & 2012 & 03 & 9,68 & 6,60 & 70 & 10,03 & 4,48 \\
\hline & 2013 & 03 & 9,68 & 6,56 & 81 & 11,60 & 5,12 \\
\hline & 2014 & 06 & 19,35 & 13,02 & 87 & 12,46 & 5,42 \\
\hline & 2015 & 05 & 16,13 & 10,77 & 94 & 13,47 & 5,77 \\
\hline & 2016 & 02 & 6,45 & 4,28 & 89 & 12,75 & 5,39 \\
\hline & 2017 & 03 & 9,68 & 6,38 & 74 & 10,60 & 4,42 \\
\hline & 2018 & 05 & 16,13 & 10,56 & 98 & 14,04 & 5,78 \\
\hline & 2019 & 04 & 12,90 & 8,39 & 105 & 15,04 & 6,11 \\
\hline & $\operatorname{VPR}^{1}(\%)$ & - & - & 27,12 & - & - & 36,38 \\
\hline \multirow{11}{*}{ 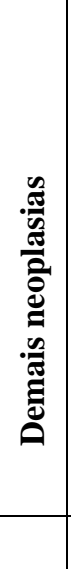 } & & \multicolumn{3}{|c|}{ Cáceres } & \multicolumn{3}{|c|}{ Mato Grosso } \\
\hline & Ano & $\mathbf{N}$ & $\%$ & Taxa & $\mathbf{N}$ & $\%$ & Taxa \\
\hline & 2012 & 22 & 8,91 & 48,41 & 620 & 11,02 & 39,71 \\
\hline & 2013 & 24 & 9,72 & 52,45 & 631 & 11,21 & 39,87 \\
\hline & 2014 & 28 & 11,34 & 60,76 & 632 & 11,23 & 39,37 \\
\hline & 2015 & 32 & 12,96 & 68,93 & 713 & 12,67 & 43,78 \\
\hline & 2016 & 40 & 16,19 & 85,62 & 719 & 12,78 & 43,56 \\
\hline & 2017 & 40 & 16,19 & 85,04 & 787 & 13,99 & 47,05 \\
\hline & 2018 & 29 & 11,74 & 61,23 & 763 & 13,56 & 45,01 \\
\hline & 2019 & 32 & 12,96 & 67,12 & 760 & 13,51 & 44,25 \\
\hline & $\operatorname{VPR}^{1}(\%)$ & - & - & 38,65 & - & - & 11,43 \\
\hline
\end{tabular}

1VPR: Variação Percentual Relativa. Fonte: Autores (2021).

Os modelos lineares apresentaram razoável qualidade de ajustes. A tendência temporal das taxas de mortalidade por câncer de colo uterino em Cáceres e na Unidade Federativa foram lineares e estáveis, assim como para as demais neoplasias no município. Apesar das taxas terem se apresentado oscilantes e crescentes durante o período estudado, elas não foram significativas $(\mathrm{p}>0,05)$, exceto para as demais neoplasias no estado de Mato Grosso que apresentou tendência linear e significativamente crescente com força de inclinação de $7 \%$ ao ano (Figura 1). 
Figura 1. Coeficiente da tendência linear da taxa de mortalidade por neoplasia de colo uterino e demais neoplasias (100 mil hab.) no município Cáceres e estado de Mato Grosso, 2012 a 2019.

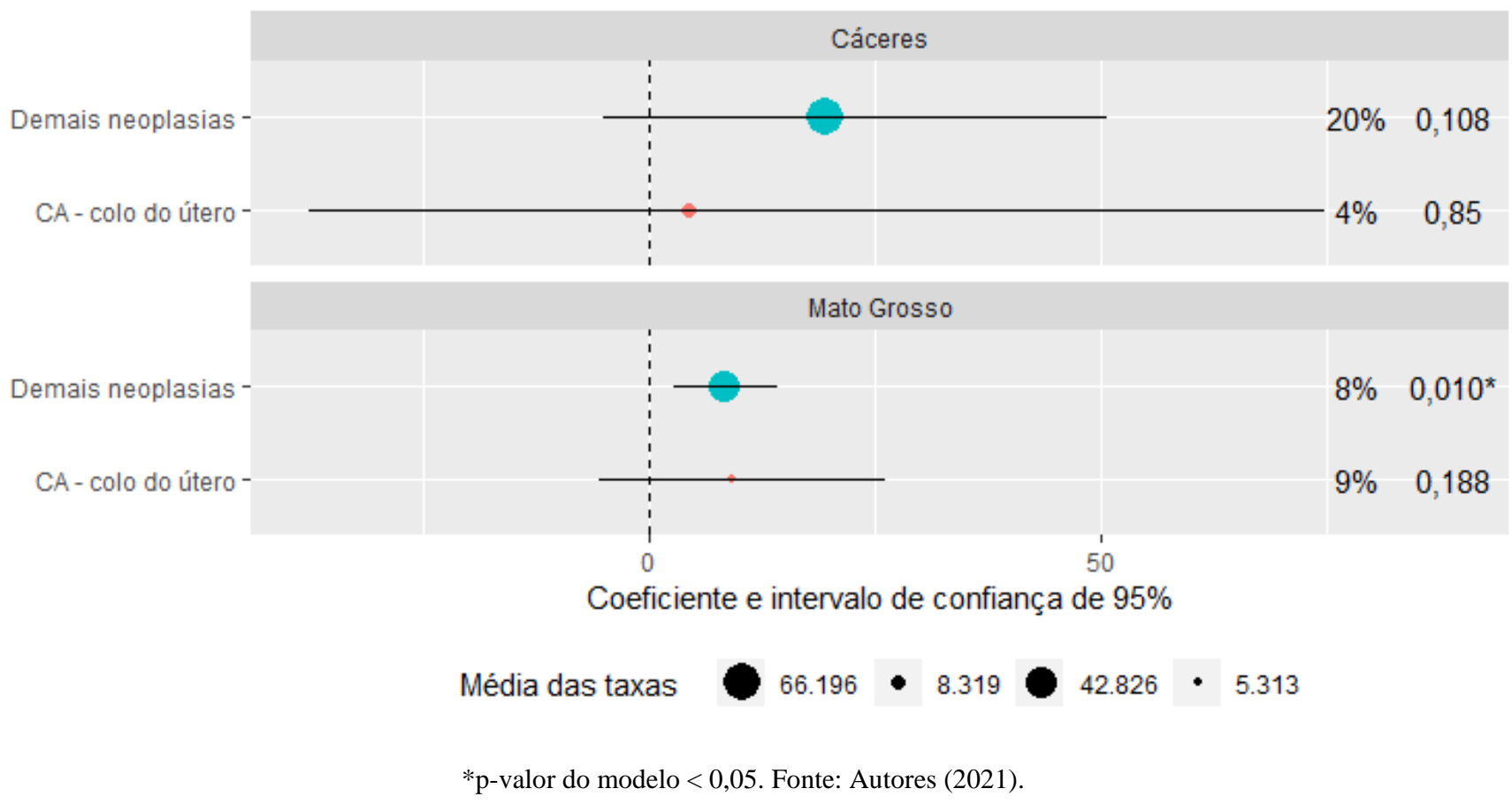

A Figura 2 apresenta a tendência da mortalidade por câncer do colo do útero e as demais neoplasias no município de Cáceres e no estado de Mato Grosso, observa-se que a tendência das taxas para o município de Cáceres se manteve superiores quando comparada a do estado em toda série.

Figura 2. Gráficos de tendência das taxas de mortalidade por neoplasias (100 mil hab.) no município Cáceres e no estado de Mato Grosso, 2012 a 2019.
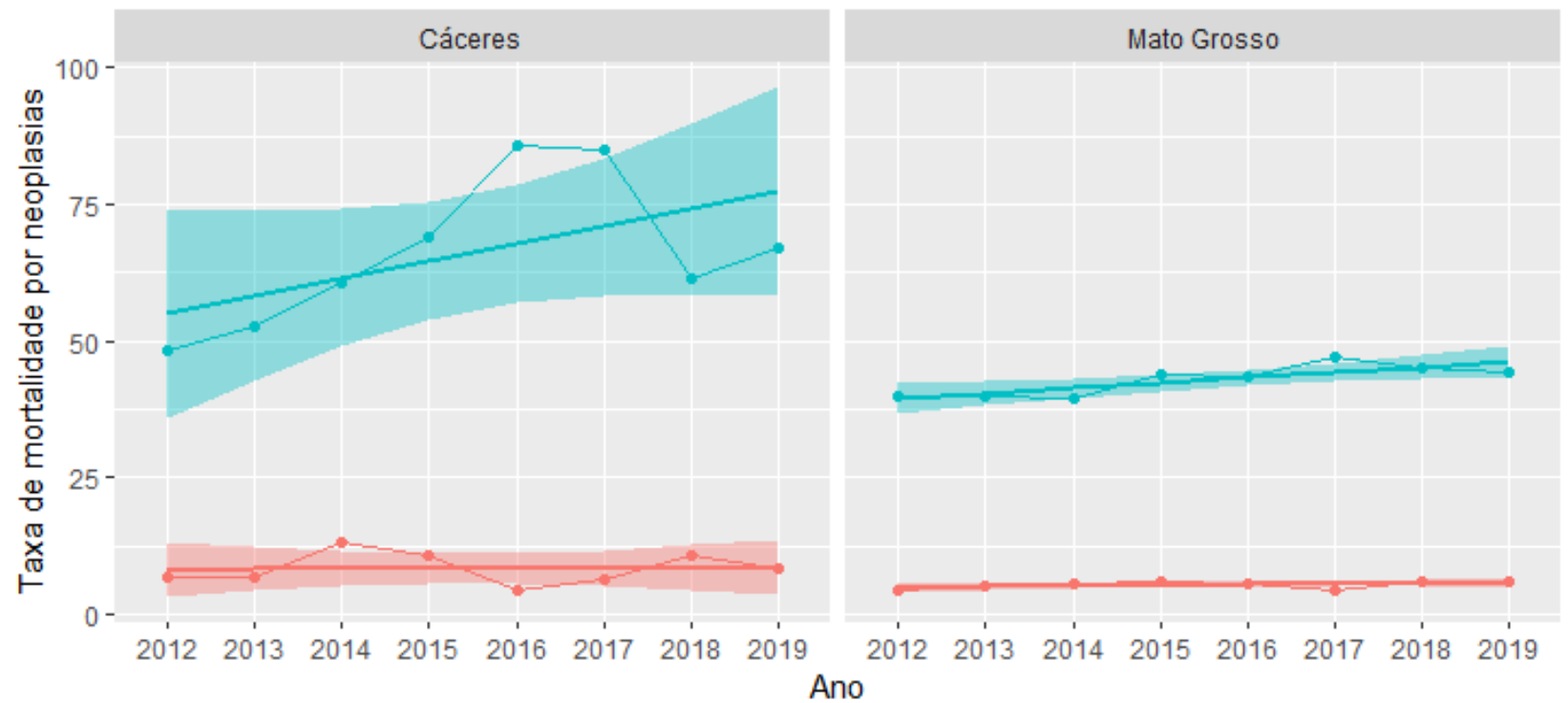

Neoplasia $\rightarrow$ CA - colo do útero $\rightarrow$ Demais Neoplasias

Fonte: Autores (2021). 


\section{Discussão}

Os resultados dessa pesquisa possibilitaram o levantamento do perfil de mortalidade por câncer do colo do útero no município de Cáceres-MT, sendo identificado a maior incidência de óbitos em mulheres pardas na faixa etária de 60 a 69 anos.

No Brasil a estratégia amplamente adotada para o rastreio do câncer do colo de útero é a realização periódica do exame citopatológico (CCO). Estudos têm demonstrado a importância da realização desse exame na redução das taxas de mortalidade ao evidenciar que, em países com cobertura de 50\% do CCO realizado periodicamente a cada três ou cinco anos apresentam taxas inferiores a 3 mortes a cada 100 mil mulheres por ano (INCA, 2016). Oliveira et al. (2018) ao estudar a respeito da cobertura de exame citopatológico em mulheres de 25 a 64 anos no Brasil, identificou que a cobertura do exame no país encontra-se abaixo da meta de $85 \%$. O autor também observou que houve menor prevalência da realização do CCO em mulheres com pouca ou nenhuma escolaridade, enquanto, mulheres com ensino superior completo apresentam a maior prevalência da realização do exame, destacando assim a necessidade da maior difusão de informação de fácil acesso e compreensão do público-alvo (Oliveira et al., 2018).

Com relação a faixa etária os resultados encontrados nesta pesquisa são similares ao de outros autores em várias regiões brasileiras (Salazar et al., 2011; Colonelli, \& Lorente, 2016; Damacena et al., 2017; Cruz et al., 2020; Silva et al., 2018; Tallon et al., 2020), que observaram que as taxas de mortalidade por câncer do colo do útero crescem a medida do avanço da idade, $\log$ a as faixas etárias inferiores a 25 anos (menor faixa etária preconizada pelo Ministério da Saúde para coleta de exame citopatológico) apresentam as menores taxas de mortalidade. Segundo a OMS (WHO, 2020), a incidência do câncer do colo do útero tem progressões crescentes a partir dos 30 anos, atingindo o pico de incidência entre 50 e 60 anos. Já a faixa etária com menor detecção de casos, Colonelli e Lorente (2016) afirmam que pacientes abaixo dos 20 anos são menos suscetíveis e representam apenas $1 \%$ dos casos de câncer do colo do útero. Acredita-se que, em mulheres com menos de 25 anos, as lesões provocadas pelo HPV serem de baixo grau e na maioria das vezes com regressão espontânea, portanto, podem ser acompanhadas clinicamente (INCA, 2011).

Em um estudo recente realizado por Martins et al. (2021), foi observado o maior percentual de óbitos na raça/cor parda chegando a representar 51,49\% dos óbitos por câncer do colo do útero em Sergipe. O autor ainda destaca que esse resultado pode ser justificado pela influência das diferenças étnicas encontradas no Brasil e todo o contexto de desigualdades e vulnerabilidade social do país. No mesmo sentido, Mendonça et al. (2008), afirma que não há uma explicação biológica para justificar a alta taxa de mortalidade por câncer do colo do útero em mulheres negras/pardas, e atribui esse fator a influência dos fatores socioeconômicos dessa população, nesse sentido, a baixa escolaridade, baixa cobertura do exame preventivo, início precoce da atividade sexual, multiparidade, longas jornadas de trabalho, burocracias do acesso aos serviços de rastreio do câncer do colo do útero no Sistema Único de Saúde (SUS) e a falta de conhecimento sobre a doença, são fatores de risco para o desenvolvimento da doença (Costa et al., 2011; Lima et al., 2006; Carvalho et al., 2018; Lobo et al., 2018; Lopes \& Ribeiro, 2019).

Referente a variável raça/cor em anos anteriores, vários estudos apontaram uma mudança nos índices para ocorrência de câncer do colo do útero. Os achados de Feres et al. (2018) e Fonseca et al. (2021) evidenciaram que, até meados de 2013 apresentavam maior ocorrência em mulheres brancas, porém, passou a ser mais frequente em mulheres pretas e pardas em anos subsequentes. Contudo, Feres et al. (2018) enfatiza que os dados relacionados a raça não são muito confiáveis devido ao Brasil ser um país com muitas misturas étnicas.

Os dados desse estudo evidenciaram tendência de estabilidade nas taxas de mortalidade por câncer do colo do útero ao longo dos anos de 2012 a 2019 em Cáceres-MT, a tendência estável não representa taxa baixas, e sim que não ocorreu alterações significativas ao longo dos 08 anos de estudo, ressaltasse se que o município apresenta taxas superiores ao do estado durante o período, esses resultados podem estar relacionados com a implementação de planos e diretrizes governamentais 
voltados ao enfrentamento de doenças crônicas, como o Plano de ações estratégicos para o enfrentamento das Doenças Crônicas não transmissíveis (DCNT) no Brasil (2011-2022) que também aborda ações voltadas a saúde da mulher e medidas profiláticas ao câncer de mama e do colo do útero, principalmente na atenção primaria à saúde (Brasil, 2013).

Para a OMS, o controle abrangente do câncer cervical inclui a prevenção primária (vacinação contra HPV), prevenção secundária (rastreamento e tratamento de lesões pré-oncológicas), prevenção terciária (diagnóstico e tratamento do câncer de colo uterino invasivo) e cuidados paliativos (WHO, 2020). O caderno de Atenção Básica $n^{\circ} 13$ publicado pelo Ministério da Saúde em 2013, é voltado ao controle dos cânceres do colo do útero e da mama, sendo nele encontrado a descrição da linha de cuidado do câncer do colo do útero e suas diretrizes que englobam: prevenção e detecção precoce do câncer do colo do útero, programa nacional de qualidade da citologia, acesso à confirmação diagnóstica para tratamento precoce das lesões percussoras do câncer do colo uterino, e tratamento adequado (Brasil, 2013).

Conforme a OMS (WHO, 2007), no caso do câncer do colo do útero, mesmo que haja vacinação contra o vírus do HPV amplamente introduzido para mulheres jovens antes da infecção, os efeitos satisfatórios da vacina serão vistos apenas quando essas jovens atingirem a idade aproximada de 50 anos. Desse modo, os programas de detecção precoce do câncer do colo do útero continuam sendo a estratégia de melhor resposta a curto prazo para a prevenção da doença, bem como para a redução de taxa de mortalidade, pelo menos nos próximos 30 anos.

\section{Conclusão}

A partir deste estudo identificou-se que no município de Cáceres os óbitos por câncer do colo do útero são mais frequentes em mulheres pardas na faixa etária de 60 a 69 anos. De modo geral, houve tendência estável para taxa de mortalidade ao longo dos anos estudados em Cáceres, sem alterações estatisticamente significativas.

Os dados encontrados evidencia a importância da implementação de planos governamentais que visam ações de prevenção ao câncer de colo do útero, considerando que a educação em saúde, o rastreamento e diagnóstico precoce da doença são as principais ferramentas utilizadas na prevenção do câncer do colo do útero no Brasil. Dentre as estratégias que podem ser realizadas, está a divulgação de informações sobre a temática nos meios de comunicação e a instauração de medidas de prevenção e educativas sobre a temática.

Contudo, ressalta-se a importância de pesquisas nesta área visando melhorias nos cuidados prestados, uma vez que a partir dos resultados encontrados, pode-se perceber que o controle do câncer do colo do útero ainda é um desafio, demonstrando a necessidade de melhorias nos programas de prevenção.

\section{Referências}

Alves, C. M. M., Guerra, M. R., \& Bastos, R. R. (2009). Tendência de mortalidade por câncer de colo de útero para o Estado de Minas Gerais, Brasil, 19802005. Cadernos de Saúde Pública, 25(8), 1693-1700. https://doi.org/10.1590/s0102-311x2009000800005

Antunes, J. L. F., \& Cardoso, M. R. A. (2015). Uso da análise de séries temporais em estudos epidemiológicos. Epidemiologia e Serviços de Saúde, 24(3), 565-576. https://doi.org/10.5123/s1679-49742015000300024

Brasil. (2013). Controle dos cânceres do colo do útero e da mama. (2a ed.), Ministério da Saúde.

Carvalho, P.G., O'dwer, G., \& Rodrigues, N. C. P. (2018). Trajetórias assistenciais de mulheres entre diagnóstico e início de tratamento do câncer de colo uterino. Saúde Debate, 42 (118), 687-701.

Colonelli, D. E., \& Lorente, S. (2016). Frequência das lesões detectadas no exame citopatológico, distribuídas por faixa etária, em mulheres atendidas na região do Vale do Ribeira, entre 2014 e 2015. Boletim Epidemiológico Paulista, 13(155), 1-10.

Costa, J. H. G., Souza, I. R. A. S., Santos, E. J. A. S., Prazeres, B. A. P., Andrade, M. L., Melo, M. F. C., Tsutsumi, M. Y., \& Sousa, M. S. (2011) Prevenção do câncer de colo do útero em comunidades ribeirinhas atendidas pelo programa Luz a Amazônia, estado do Pará, Brasil. Revista Pan-Amazônica de Saúde, 2(4), 17-22.

Cruz, N. M. R. A. da, Cruz, K. E. A. da, \& Silva, C. A. L. da. (2020). Mortalidade Por Câncer Do Colo Do Útero No Estado Da Bahia, Brasil, Entre 1996 E 2012. Revista Baiana de Saúde Pública, 42(4), 624-639. https://doi.org/10.22278/2318-2660.2018.v42.n4.a2807 
Damacena, A. M., Luz, L. L., \& Mattos, I. E. (2017) Rastreamento do câncer do colo do útero em Teresina, Piauí: estudo avaliativo dos dados do Sistema de Informação do câncer do colo do útero, 2006-2013. Epidemiologia e Serviços de Saúde, 26(1), 71-80.

DATASUS. (2021a). Projeção da População das Unidades da Federação por sexo e grupos de idade: 2000-2030. http://tabnet.datasus.gov.br/cgi/tabcgi.exe?ibge/cnv/projpopuf.def.

DATASUS. (2021b). Sistema de Informações sobre Mortalidade. TabNet Win32 3.0 : Mortalidade-Brasil. http://tabnet.datasus.gov.br/cgi/deftohtm.exe?sim/cnv/obt10uf.def

Feres, T. M., Rodrigues, L. S., Coser, E. X., Ferreira, G. J., Murer, L. A. M., Campos, T. G., \& Rocha, L. L. V. (2018). Prevalência de câncer no colo uterino: um estudo descritivo. Brasilian Journal of Surgery and Clinical Research, 22(2), 54-58.

Ferlay, J., Soerjomataram, I., Dikshit, R., Eser, S., Mathers, C., Rebelo, M., Parkin, D. M., Forman, D., \& Bray, F. (2015). Cancer incidence and mortality worldwide: Sources, methods and major patterns in GLOBOCAN 2012. International Journal of Cancer, 136(5), E359-E386 https://doi.org/10.1002/ijc.29210.

Fonseca, T. A. A., Silva, D. T. A., \& Silva, M. T. A. (2021). Distribuição dos óbitos por cânceres de colo do útero no Brasil. Journal of Health \& Biological Sciences, 9(1), 1-6.

INCA (2011). Diretrizes brasileiras para o rastreamento do câncer do colo do útero. (2a ed.).

INCA (2021a). Conceito e Magnitude. https://www.inca.gov.br/controle-do-cancer-do-colo-do-utero/conceito-e-magnitude.

INCA (2021b). Câncer do colo do útero. https://www.inca.gov.br/tipos-de-cancer/cancer-do-colo-do-utero.

Lima, C. A., Palmeira, J. A. V., \& Cipolotti, R. (2006). Fatores associados ao câncer do colo uterino em Propriá, Sergipe, Brasil. Caderno de Saúde Pública, 22(10), 2151-2156.

Lobo, L. M. G. A., Almeida, M. M., \& Oliveira, F. B. M. (2018). Câncer do colo uterino, HPV e exame papanicolau: uma reflexão acerca dos conhecimentos das mulheres. Revista Ciência \& Saberes, 4(1), 889-895.

Lopes, V. A. S., \& Ribeiro, J. M. (2019). Fatores limitadores e facilitadores para o controle do câncer de colo de útero: uma revisão de literatura. Ciência \& Saúde Coletiva, 24(9), 3431-3442.

Martins, M. C. V., Carregosa, K. R. S., Anchieta, M. C. M., Batista, J. F. C., Andrade, T. R. S. F., Santos, I. H. A., Ferrari, Y. A. C., Jesus, C. V. F., Góis, R. M. O., Galotti, F. C. M., \& Lima, S. O. (2021). Tendência temporal da mortalidade por câncer do colo do útero em Sergipe. Research, Society and Development, 10(1), 1-14.

Mendonça, V. G., Lorenzato, F. R. B., Mendonça, J. G., Menezes, T. C., \& Guimarães, M. J. B. (2008). Mortalidade por câncer do colo do útero: características sociodemográficas das mulheres residentes na cidade de Recife, Pernambuco. Revista Brasileira de Ginecologia e Obstetrícia, 30(5), 248-255.

PAHO (2016). Controle integral do câncer do colo do útero. Guia de práticas essenciais. OPAS.

PAHO (2018). Elementos conceituais e práticos. OPAS

RIPSA (2008). Indicadores Básicos Para a Saúde No Brasil: Conceitos e Aplicações 2.ed. http://tabnet.datasus.gov.br/tabdata/livroidb/2ed/indicadores.pdf

Salazar, M. A. A., Souz, M. L., Martins, H. E. L., Locks, M. T. R., Monticelli, M., \& Peixoto, H. G. (2011). Câncer do colo do útero: mortalidade em Santa Catarina-Brasil, 2000 a 2009. Texto \& Contexto Enfermagem, 20(3), 541-546.

Silva, R. C. G., Silva, A. C. O., Peres, A. L., \& Oliveira, S. R. (2018). Perfil de mulheres com câncer do colo do útero atendidas para tratamento em centro de oncologia. Revista Brasileira de Saúde Materno Infantil, 18(4), 703-710.

Silva, G. A. e, Noronha, C. P., Santos, M. de O., \& Oliveira, J. F. P. (2008). Diferenças de gênero na tendência de mortalidade por câncer de pulmão nas macrorregiões brasileiras. Revista Brasileira de Epidemiologia, 11(3), 411-419. https://doi.org/10.1590/s1415-790x2008000300008

Tallon, B., Monteiro, D., Soares, L., Rodrigues, N., \& Morgado, F. (2020). Tendências da mortalidade por câncer de colo no Brasil em 5 anos (2012-2016). Saúde e Debate, 44(125):362-371.

WHO (2007). Cancer Control: Knowledge into Action: WHO Guide for Effective Programmes. World Health Organization.

WHO (2020). Human papillomavirus (HPV) and cervical cancer. https://www.who.int/news-room/fact-sheets/detail/human-papillomavirus-(hpv)-andcervical-cancer. 